\title{
Extraction and physicochemical characterization of chitin and chitosan from Zophobas morio larvae in varying sodium hydroxide concentration
}

\begin{abstract}
Large amount of sodium hydroxide $(\mathrm{NaOH})$ is consumed to remove the protein content in chitin biomass during deproteinization. However, excessive $\mathrm{NaOH}$ concentration used might lead to the reduction of cost effectiveness during chitin extraction. Hence, the present study aimed to extract and evaluate the physicochemical properties of chitin and chitosan isolated from superworm (Zophobas morio) larvae using 0.5 M-2.0 M of $\mathrm{NaOH}$. The extracted chitin and chitosan were subjected to Fourier Transform Infrared Spectroscopy (FT-IR), elemental analysis, Scanning Electron Microscope (SEM), Thermogravimetric Analysis (TGA), Differential Scanning Calorimetry (DSC) and X-ray Diffraction (XRD). The 0.5 M NaOH treatment resulted in the highest yield of chitin (5.43\%), but produced the lowest yield (65.84\%) of chitosan. The extracted chitin samples had relatively high degree of acetylation (DA) (82.39\%-101.39\%). Both chitin and chitosan showed smooth surface with tiny pores. The extracted chitin samples were confirmed as $\alpha$-chitin based on the FT-IR and TGA. The chitin samples were amorphous with low degree of crystallinity. From TGA, the Chitosan 3 extracted was partially deacetylated. Both DPPH radical scavenging and ferric-chelating assay showed positive correlation with DD of chitosan isolates. However, the chitosan isolates were not fully dissolved, resulting in lower radical scavenging and ferric-chelating ability compared to commercial chitosan.
\end{abstract}

Keyword: Chitin; Chitosan; Zophobas morio; Physicochemical properties 